\title{
ARTIGOS
}

\section{ESTUDO DA VIABILIDADE DE FABRICAÇÃO DE FILAMENTO COMPÓSITO SUSTENTÁVEL PARA IMPRESSÃO 3D A PARTIR DE UMA MATRIZ PLA REFORÇADA COM RESÍDUOS DE FIBRAS DE MADEIRA}

\section{FEASIBILITY STUDY FOR THE PRODUCTION OF SUSTAINABLE COMPOSITE FILAMENT FOR 3D PRINTING FROM A PLA MATRIX REINFORCED WITH WOOD FIBER RESIDUES}

\author{
DANIEL LAUXEN SPOHR | UNISINOS \\ FELIPE ANTÔNIO LUCCA SÁNCHEZ, Dr. | UNISINOS \\ ANDRÉ CANAL MARQUES, Dr. | UNISINOS
}

\begin{abstract}
RESUMO
Processos tecnológicos de manufatura aditiva, como a de impressão 3D, vem permitindo transcender os limites do desenvolvimento dos produtos atuais a um custo menor em de forma mais rápida. Nos últimos anos a impressão 3D se tornou mais fácil de usar e mais barata, tornando acessível para diferentes mercados. A literatura vem demonstrando que há uma grande demanda para conceber materiais mais sustentáveis para fabricação de bens de consumo por impressão 3D o que inclui, por exemplo, a utilização de resíduos de processos industriais da indústria moveleira como pós e serragens incorporados a matrizes poliméricas. Neste trabalho, foi utilizada a combinação de PLA com resíduos de pó de madeira com objetivo de criar um filamento com aspecto de madeira, com propriedades mecânicas melhores do que o filamento sem a adição da carga e assim mais sustentável. Para isto, fez-se primeiramente a caracterização do pó de madeira para determinar os melhores parâmetros de processo e após foram determinadas três formulações diferentes e extrudadas para a produção dos filamentos. Ao final foram analisadas quanto a suas propriedades de interesse.
\end{abstract}

PALAVRAS CHAVE: Manufatura aditiva; Filamentos; Sustentabilidade

\begin{abstract}
Technological processes of additive manufacturing, such as 3D printing, allowed to transcend the limits of the development of current products at a lower cost in a faster way. In recent years, 3D printing has become easier to use and cheaper, making it accessible to different markets. The literature has shown that there is a great demand to design more sustainable materials for the manufacture of consumer goods by 3D printing, which includes, for example, the use of industrial process residues from the furniture industry as powders and sawdust incorporated into polymer matrices. In this work, the combination of PLA with wood dust residues was used in order to create a wood-like filament, with better mechanical properties than the filament without the addition of the load and thus more sustainable. For this, the characterization of the wood powder was done first to determine the best process parameters and then three different and extruded formulations were determined for the production of the filaments. At the end, they were analyzed for their properties of interest.
\end{abstract}

KEY WORDS: Additive manufacturing; Filaments; Sustainability 


\section{INTRODUÇÃO}

A manufatura aditiva ou impressão 3D é uma tecnologia que permite transcender os limites do desenvolvimento de produto atuais a um custo menor em menos tempo. Desde protótipos funcionais até peças prontas, a impressão 3D oferece uma variedade de solução em inúmeras aplicações. Nos últimos anos a impressão 3D se tornou mais fácil de usar e mais barata, tornando acessível para diferentes mercados.

Por outro lado, as demandas por tecnologias e práticas mais sustentáveis são cada vez mais eminentes e é consenso que é preciso mitigar o uso de matérias primas de procedência não renovável. O poliácido lático (PLA) desponta como um polímero de engenharia com amplas aplicações que pode ser obtido a partir de recursos renováveis como do amido de milho e o seu uso na forma de filamentos para a impressão 3D tem crescido significativamente nos últimos anos. Existe grande interesse na impressão 3D de materiais mais sustentáveis, o que inclui a utilização de compósitos que incorporem, reciclando, resíduos industriais. Dessa maneira, neste trabalho, foi utilizada a combinação de PLA com diferentes teores de resíduos de pó de madeira da indústria moveleira local. Assim, o principal objetivo deste trabalho foi a produção de um filamento compósito baseado em uma matriz PLA e reforçado com diferentes teores fibras de madeira para avaliação da viabilidade na impressão 3D de artefatos.

\section{REVISÃO DA LITERATURA}

Apresenta-se aqui os principais tópicos de revisão de literatura.

\subsection{Manufatura aditiva}

Impressão 3D ou manufatura aditiva é um processo que fabrica produtos através da deposição sucessiva de camadas de material (CHIA, 2015). Com esta tecnologia, primeiramente um modelo digital é gerado usando um software CAD (Computer Aided Design) e então impresso a partir de matérias primas na forma de granulados, pós, fluídos com viscosidade controlada dependendo da tecnologia utilizada. A tecnologia traz inúmeras oportunidades uma vez que tudo o que está na forma digital pode ser impresso, isto inclui artigos decorativos para casa, peças mecânicas, calçados etc. (SCHUBERT, 2014).

Ainda mais surpreendente é a liberdade de design das peças impressas. Imprime-se geometrias antes improváveis com os métodos tradicionais de manufatura além de ser possível integrar partes das peças que antes seria necessário serem produzidas separadamente e depois montadas usando adesivos ou parafusos (ZHONG, 2017).
Além disso, um ponto chave para a adoção da manufatura pelas empresas é a disponibilidade de materiais com características específicas. Por isto esta área vem atraindo atenção da comunidade científica que não tem medido esforços para o aperfeiçoamento da manufatura aditiva e de suas matérias primas. Levando em consideração estas características da tecnologia, entende-se que a manufatura aditiva é ponto chave no contexto da indústria 4.0 para a transformação digital das empresas (GREENE, 2017).

\subsection{Filamentos sustentáveis}

A tecnologia FFF (Fused Filament Fabrication), também conhecido como FDM (Fused Deposition Modeling) é a tecnologia de impressão 3D mais utilizada. FFF usa polímeros termoplásticos, que vem em forma de filamento. $O$ filamento é fundido e pressionado através de um bico de extrusão. A impressora movimenta o bico, depositando material fundido em locais precisos, seguindo um caminho pré-determinado pelo software da máquina. Quando o material sai do bico de extrusão e chega na plataforma de impressão, ele resfria e se solidifica. Desta forma as peças são criadas camada por camada (WANGA, 2017).

$\mathrm{O}$ aumento da preocupação com temas ambientais, como aquecimento global tem renovado o interesse em materiais poliméricos mais ecológicos do que os derivados do petróleo. O PLA, poliácido láctico é um material biodegradável produzido por um monômero derivado do amido de milho, satisfaz os critérios ambientais e possui características que permitem a substituição dos materiais usuais derivados do petróleo. PLA possui boa resistência mecânica o que o torna versátil para uma grande grama de aplicações, como sacolas plásticas, embalagens, copos, peças simples etc. No entanto, a utilização mais ampla do PLA é restringida pela sua baixa resistência ao impacto bem como o preço elevado se comparado com polietileno e polipropileno. Compósitos que incorporam fibras naturais resultam em materiais com melhores propriedades mecânicas, o que pode ser interessante no caso do PLA. O benefício de se optar por fibras naturais ao invés dos agentes de reforço convencionais como fibra de vidro, talco ou fibra de carbono incluem baixa densidade e o fato de agregar o conceito de sustentabilidade ao material. Ainda, as fibras de origem natural geralmente são mais baratas e muitas vezes podem ser obtidas do aproveitamento de resíduos fabris, como a serragem que é um subproduto da indústria madeireira.

A madeira é um material orgânico e natural que está amplamente disponível na forma de resíduo e pode ser facilmente triturado em frações menores, até chegar ao 
estado de pó (LE DUIGOUA et al., 2016). O efeito do pó da madeira no compósito depende da sua propriedade, da distribuição de tamanho de partícula e da compatibilidade com a matriz polimérica, que configura no principal desafio deste tipo de material (TAO, 2017).

Existe grande interesse na impressão 3D de materiais mais sustentáveis, o que inclui a utilização de materiais reciclados, compósitos com madeira, celulose, casca de cacau etc. (BARBOSA, 2011). Existem inúmeros tipos de fibras naturais já testadas em matrizes poliméricas como bamboo, juta, algodão, papel, sisal e celulose. A interação entre estas partículas naturais e o polímero influenciam totalmente as propriedades mecânicas do compósito final. Estudos mostram que no caso do PLA, compostos naturais contendo celulose, como a madeira, aumentam o modulo elástico e diminuem a resistência a tração do material. Este fato se deve a fraca afinidade química entre matriz e carga, uma vez que o PLA é apolar e a madeira é fortemente polar. Mesmo assim, com controles de processo ajustados, é possível criar compostos homogêneos e de boa resposta para aplicações onde não é exigida resistência a tração (BARBOSA, 2011).

\section{PROCEDIMENTOS METODOLÓGICOS}

Para o desenvolvimento de filamentos compósitos de PLA com pó de madeira neste trabalho de pesquisa, o procedimento experimental foi dividido em quatro etapas principais que incluem: Caracterização do pó de madeira; Formulação e fabricação dos compósitos por extrusão; Caracterização dos filamentos produzidos; Teste de impressão 3D.

\subsection{Materiais}

Foram utilizados basicamente dos materiais: resíduos de pós de madeira de processos de beneficiamento de madeiras da indústria moveleira e pellets de PLA virgem como matriz termoplástica para formulação dos diferentes compósitos. A amostra de pó de madeira é um resíduo oriundo do processo de usinagem de uma fábrica de móveis. É uma mistura das seguintes espécies: Tectona grandis, conhecida como Teca, Melia azedarach, conhecida como Cinamomo, Couratari spp, conhecida como Tauari e o Eucalyptus globulus conhecido como Eucalipto comum. Trata-se de um resíduo irregular que contém pedaços grosseiros de madeira. Portanto, foi necessário beneficiá-lo para utilizá-lo como carga do filamento, sendo submetido à moagem. Foi colocado, em cada moinho de bolas, 200 gramas de material e utilizado nove esferas, com tempo de permanência de 30 minutos e 100 rpm de rotação. Depois deste processo, o resíduo foi passado em peneira com abertura de 300 micrometros. O material que passasse nas aberturas da peneira era separado e classificado como pó de madeira. O restante voltava ao moinho para continuar o processo de moagem.

O material PLA utilizado foi o PLA 2003d em forma de pellets da marca Nature Works (2019). Trata-se de um polímero transparente próprio para extrusão que pode ser usado naturalmente ou como parte de uma blenda. É um biopolímero de alto peso molecular que pode ser processado por extrusora convencional. Na tabela 1 abaixo são descritos os dados técnicos do material. Estes valores são fornecidos pelo fabricante.

\begin{tabular}{|l|l|l|}
\hline Propriedades & Valores & Método ASTM \\
\hline Densidade & $1,24 \mathrm{~g} / \mathrm{cm}^{3}$ & $\mathrm{D} 792$ \\
\hline Índice de fluidez & $6 \mathrm{~g} / 10 \mathrm{~min}$ & $\mathrm{D} 1238$ \\
\hline $\begin{array}{l}\text { Resistência a } \\
\text { tração até ruptura }\end{array}$ & $53 \mathrm{Mpa}$ & $\mathrm{D} 882$ \\
\hline $\begin{array}{l}\text { Limite de } \\
\text { escoamento }\end{array}$ & $60 \mathrm{Mpa}$ & $\mathrm{D} 882$ \\
\hline $\begin{array}{l}\text { Módulo de } \\
\text { elasticidade }\end{array}$ & $3.5 \mathrm{Mpa}$ & $\mathrm{D} 882$ \\
\hline Alongamento & $6 \%$ & $\mathrm{D} 882$ \\
\hline $\begin{array}{l}\text { Temperatura } \\
\text { de Extrusão }\end{array}$ & $180-190^{\circ} \mathrm{C}$ & - \\
\hline
\end{tabular}

Tabela 1: Ficha técnica do PLA 2003d. Fonte: Nature Works (2019).

\subsection{Caracterização do pó de madeira}

Tendo em vista que o estudo propõe o uso do pó de madeira como carga de reforço para extrusão de filamentos para uso em impressão 3D foram levantadas as características físico-químicas de interesse, que tangem o tamanho das partículas, natureza química, aspecto e forma física e higroscopicidade. Nesse artigo são relatadas algumas dessas análises que incluem o teor de umidade, a densidade, a distribuição granulométrica, a área superficial específica por adsorção de N2 e uma análise termogravimétrica do resíduo de madeira usado na fabricação dos compósitos.

\subsubsection{Teor de umidade}

A determinação do teor de umidade da amostra foi realizada no Laboratório de Caracterização e Valorização de Materiais da Unisinos, no instrumento Determinador de Umidade marca BEL Engineering Ltda. O aparelho é uma maneira automatizada de determinação da densidade por secagem. A amostra é pesada e posteriormente submetida à temperatura de $105^{\circ} \mathrm{C}$ até que haja estabilização. Quando a pesagem da amostra não apresenta mais alteração, entende-se que toda água foi evaporada e 
então o valor é considera. O resultado da umidade é obtido através da subtração do peso final com o peso inicial. $A$ margem de erro do aparelho é de 0,1\% (OLIVEIRA, 2009).

\subsubsection{Densidade}

A determinação da densidade da amostra foi realizada em aparelho picnômetro no Laboratório de Caracterização e Valorização de Materiais da Unisinos. A picnometria de gás Hélio tem como objetivo avaliar a densidade e volume real do material de interesse. $\mathrm{O}$ ensaio é realizado pela comparação da variação de pressão do gás Hélio entre uma câmara de volume conhecido e uma câmara com a amostra a ser analisada. Utiliza-se gás Hélio para se obter maior precisão da análise, devido a facilidade do mesmo penetrar na amostra e preencher os vazios da mesma, revelando a densidade e o volume real da amostra (FREITAS, 2014).

\subsubsection{Distribuição granulométrica}

A caracterização granulométrica da amostra foi realizada no Laboratório de Caracterização e Valorização de Materiais da Unisinos, de acordo com a norma CEMP n॰ 81 (ABIFA 2003) utilizando peneiras do sistema Tyler. O método consiste em colocar $30 \mathrm{~g}$ da amostra (seca em estufa fim. A figura 1 mostra a extrusora portátil de mesa usada para fabricação dos filamentos compósitos.

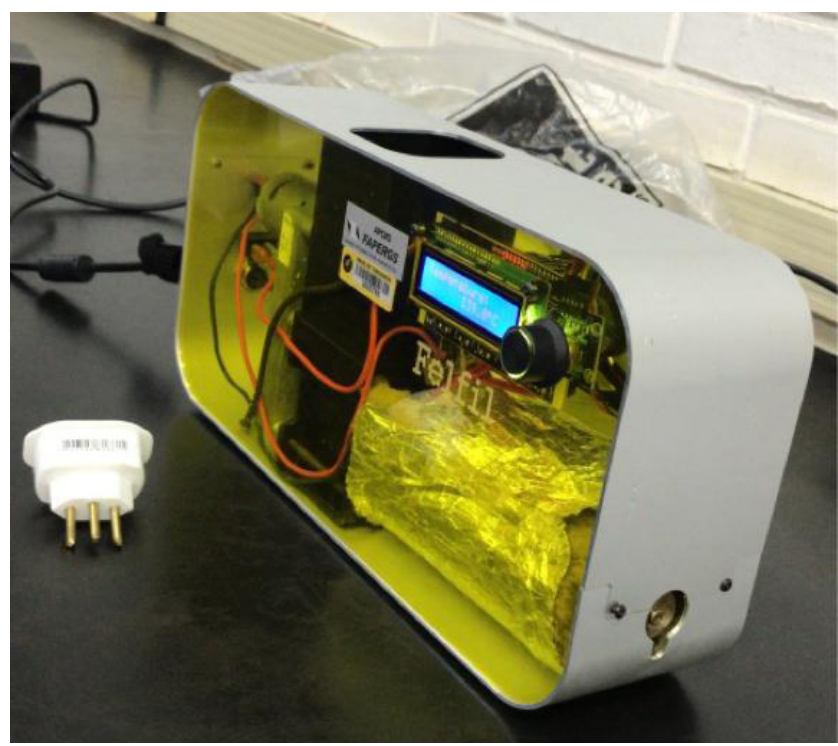

Figura 01: Extrusora Felfil utilizada para confecção de filamentos para impressão 3D. Fonte: Autores.

Para atingir um diâmetro consistente a máquina foi ajustada em relação a temperatura e velocidade da rosca. Estes parâmetros foram examinados e selecionados até que se atingiu um filamento de 1,75mm de diâmetro. Esta etapa visa extrudar três diferentes formulações de PLA com pó de madeira. As concentrações das cargas foram baseadas em um estudo de Le Duigou et al (2016), que determinou que a concentração de carga dos filamentos de madeira disponíveis no mercado varia de $10 \%$ a $20 \%$ e no estudo de Kariz (2018) que produziu filamentos de PLA com pó de madeira utilizando as mesmas concentrações. Baseado nestes dois trabalhos foram definidas as concentrações do pó de madeira nos filamentos. Portanto, a quantidade de pó de madeira adicionada foi de $0 \%, 10 \%, 15 \%$ e $20 \%$ e está descrita na tabela 2 abaixo. $O$ filamento sem adição de carga serve como amostra de controle para as posteriores análises.

\begin{tabular}{|l|l|}
\hline Nomenclatura das amostras & Composição \\
\hline PLA & $100 \%$ PLA \\
\hline PLA10M & $90 \%$ PLA E 10\% pó de madeira \\
\hline PLA15M & 85\% PLA E 15\% pó de madeira \\
\hline PLA20M & 80\% PLA E 20\% pó de madeira \\
\hline
\end{tabular}

Tabela 2: Concentração de pó de madeira na formulação.

Fonte: Autores.

\subsection{Formulação e fabricação dos compósitos por extrusão}

No presente trabalho foi utilizada uma extrusora com uma rosca de alimentação e uma zona de aquecimento da marca Felfil modelo EVO desktop própria para este
O termoplástico usado foi o PLA Ingeo ${ }^{\text {TM }}$ 2003D (NatureWorks, 2019) na forma de pellets. Os pellets foram secos durante 2 horas a $90^{\circ} \mathrm{C}$ em estufa seguindo 
recomendação do fabricante e as partículas de pó de madeira foram secas em estufa a $103^{\circ} \mathrm{C}$ graus por mais de 24 horas, para garantir completa ausência de umidade (KARIZ, 2018). Feito isto, os materiais foram pesados conforme as formulações e agitados mecanicamente. $\mathrm{O}$ cuidado com o preparo do pó de madeira se faz necessário porque mesmo partículas com aproximadamente 0,3mm, podem obstruir a saída do bico de impressão (BRENT, 2015).

Determinou-se em testes preliminares as temperaturas mais favoráveis para extrusão dos filamentos e se observou uma mudança na temperatura conforme o teor de madeira presente no compósito. Assim a temperatura de extrusão foi de $210^{\circ} \mathrm{C}$ para a formulação PLA20M, $200^{\circ} \mathrm{C}$ para a formulação $\mathrm{PLA} 15 \mathrm{M}, 190^{\circ} \mathrm{C}$ para a formulação PLA10M e $185^{\circ} \mathrm{C}$ para a formulação com o PLA puro. A rotação da rosca foi mantida em 9 rpm de modo a gerar filamentos com $1.75 \mathrm{~mm}$ de diâmetro para todas as formulações.

\subsection{Caracterização dos filamentos produzidos}

\subsubsection{Dureza}

As medições de dureza foram feitas em um durômetro digital Woltest, modelo GSD 702D, seguindo a norma ASTM 7456 obtendo-se os valores na escala Shore D, medindo-se sete pontos em cada amostra. Foi aplicada uma carga de cinco quilos durante 3 segundos de endentação (TEIXEIRA, 2006).

\subsection{2 Índice de fluidez}

A determinação do índice de fluidez é efetuada extrusando o polímero em um reômetro capilar, que consiste em um barril de extrusão aquecido, na extremidade do qual é montada uma matriz capilar de dimensões específicas. O reômetro é operado com pressão imposta, decorrente da aplicação de uma carga constante e bem definida no topo da coluna do fundido. O índice de fluidez é obtido a partir da taxa de fluxo do fluido sob imposição desta carga, e seu valor é expresso pela quantidade de material extrusado, em gramas, por 10 minutos (ROCHA, 1994). O ensaio foi realizado seguindo a norma ASTM D $12238 \mathrm{em}$ plastômetro Gotterfeld com carga de $5 \mathrm{~kg}$.

\subsubsection{Microscopia óptica}

Para esta análise foi utilizado um estéreo microscópio da marca Zeiss modelo Stemi 305. O ensaio foi feito no laboratório de Sedimentologia da UNISINOS. Na técnica utilizada, a luz é transmitida atravessando a amostra e formando a imagem ampliada. Com isto é possível ver coloração, fraturas, bolhas, vazios etc. (FERNANDES, 2016). No presente trabalho, o intuito da análise está em avaliar a dispersão do pó de madeira na matriz de PLA.

\subsubsection{Homogeneidade do filamento}

Para determinar a homogeneidade do filamento foram feitas medições do diâmetro de dois metros de filamento a cada $10 \mathrm{~cm}$. As medidas foram feitas com um paquímetro digital da marca Mitutoyo com precisão de +/- 0,03 mm.

\subsubsection{Calorimetria exploratória diferencial (CED)}

Para determinar uma possível mudança na temperatura de transição vítrea e na temperatura de fusão da matriz PLA, foi conduzida a CED e esta análise foi realizada para os filamentos compósitos, bem como para um filamento de PLA puro em calorímetro NETZSCH 404F3 do Laboratório de Biomateriais e Cerâmicas Avançadas da UFRGS, na faixa de temperatura de 25 a $350^{\circ} \mathrm{C}$ com fluxo de $\mathrm{N}_{2}$ de $20 \mathrm{~mL} / \mathrm{min}$.

\subsection{Teste de impressão 3D}

Esta etapa tem o intuito de testar as formulações quanto a sua "printabilidade", ou seja, a capacidade do filamento de imprimir peças de qualidade por impressão 3D. Para modelar o corpo de prova foi utilizado software SolidWorks e para fatiamento e parâmetros de impressão foi utilizado software Ultimaker Cura. Foi feito o teste em duas impressoras diferentes, sendo elas a Cliever CL1 e a Sethi3d S3 com condições de preenchimento de 50\% e temperatura de 225 .

\section{RESULTADOS E DISCUSSÃO}

\subsection{Caracterização do pó de madeira}

\subsubsection{Teor de umidade}

O valor encontrado de umidade no pó de madeira foi de 13,11\% indicando a importância e necessidade de uma etapa de secagem previamente ao processo de extrusão, a fim de garantir a quantidade adequada de massa de madeira e para evitar a formação de bolhas da evaporação de água nos filamentos produzidos durante a extrusão.

\subsubsection{Densidade}

Os resíduos de madeira apresentam uma variação muito grande da densidade, pois depende do tipo de madeira do resíduo e de que etapa produtiva ele é gerado. Ribeiro e Machado, 2015, determinaram a densidade de diferentes espécies de Eucalyptus e acharam valores que variam 
de 2,3 a $1,3 \mathrm{~g} / \mathrm{cm}^{3}$. Este valor está próximo ao encontrado nas amostras de pó de madeira do trabalho. $O$ valor encontrado foi de $1,38 \mathrm{~g} / \mathrm{cm}^{3}$. Mesmo assim este valor é considerado baixo tratando-se de carga de reforço e é justamente uma vantagem competitiva que as fibras naturais possuem. Em termos comparativos, a densidade do carbonato de cálcio e fibras de vidro variam entre 2,48 e 2,70 g.cm-3 respectivamente. Estudos mostram que as cargas conferem aumento na densidade dos compósitos. Sendo assim, analisar a densidade da carga é importante uma vez que tem influência direta na densidade do composto. (MORESCO, 2009)

\subsubsection{Distribuição granulométrica}

A figura 2 apresenta uma curva de distribuição granulométrica do resíduo beneficiado de madeira. É possível ver uma distribuição com característica bimodal, tendo em vista que uma grande população das partículas se encontra em duas faixas de tamanho, entre $300 \mu \mathrm{m}$ e $150 \mu \mathrm{m}$. A maior parte, $47 \%$ das partículas possuem tamanho inferior ou igual a $300 \mu \mathrm{m}$ e $30 \%$ possuem $150 \mu$ m ou menos. Com esta distribuição espera-se ter uma boa dispersão do pó de madeira na matriz polimérica, uma vez que possui partículas menores e maiores, o que facilitaria na organização e

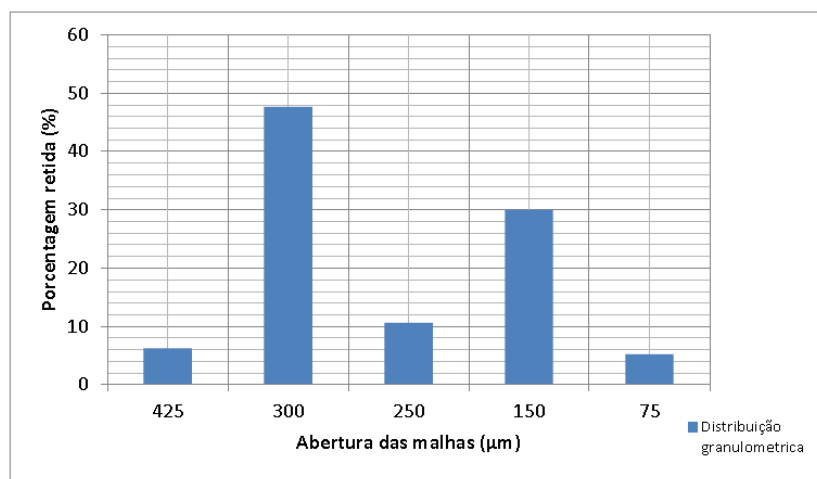

Figura 02: Distribuição granulométrica do pó de madeira Fonte: Autores.

\subsection{4 Área superficial específica}

A área superficial abrange a superfície total das partículas e seus poros. O resultado encontrado para área superficial específica, pelo método de BET foi de 0,6156 $\pm 0,0141 \mathrm{~m}^{2} / \mathrm{g}$.

\subsubsection{Termogravimetria (TGA)}

Na figura 3 abaixo é apresentado o resultado gráfico da análise termogravimétrica do pó de madeira utilizado no trabalho. Observou-se uma pequena perda de massa em temperatura próxima a $100^{\circ} \mathrm{C}$, a qual pode ser atribuída à perda de água estrutural. Depois ocorre a primeira perda de massa da amostra em torno de $240{ }^{\circ} \mathrm{C}$, devido à degradação das cadeias de hemiceluloses, celulose e uma menor degradação de parte da lignina. A segunda perda de massa ocorre em aproximadamente $350{ }^{\circ} \mathrm{C}$, devido à completa degradação da celulose e posteriormente, da lignina restante. A estabilização de massa do pó de madeira ocorreu em temperatura próxima a $540{ }^{\circ} \mathrm{C}$ quando a amostra se encontra totalmente carbonizada. Até a estabilização, houve perda de massa de aproximadamente $80 \%$.

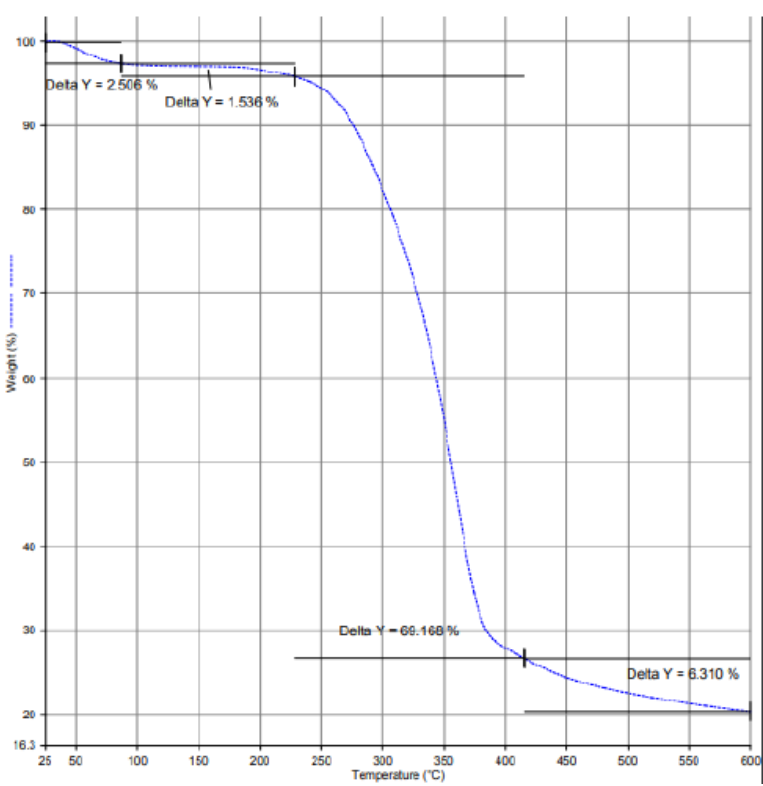

Figura 03: Análise termogravimétrica do pó de madeira usado para fabricação dos filamentos compósitos.

Fonte: Autores.

\subsection{Formulação e fabricação dos compósitos por extrusão}

O filamento utilizado no processo de manufatura aditiva por extrusão requer um diâmetro específico, além de certa flexibilidade e resistência mecânica para ser utilizado. A figura 4 mostra o filamento PLA10M produzido na extrusora de bancada.

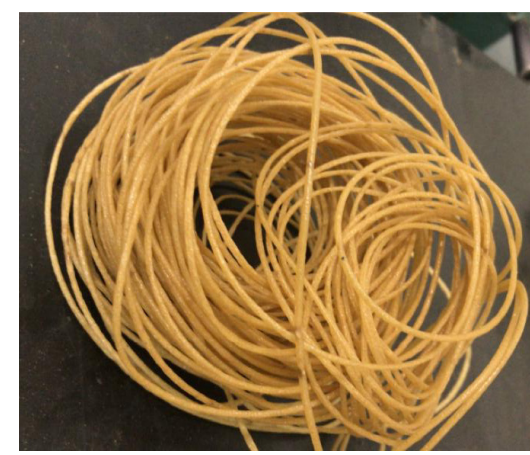

Figura 04: Filamento PLA10M de madeira produzido. Fonte: Autores. 


\subsection{Caracterização dos filamentos produzidos}

Nesta etapa, foram analisados os filamentos produzidos. Os resultados foram comparados entre si, uma vez que um dos objetivos do trabalho é selecionar a melhor concentração de pó de madeira. Além disso, fez-se a comparação dos resultados com a ficha técnica dos pellets de PLA e com ficha técnica de um material compósito de madeira comercial, a fim de validar a metodologia utilizada.

\subsubsection{Dureza}

A dureza foi utilizada como parâmetro para se inferir sobre a resistência mecânica do material. O método utilizado foi a dureza do tipo Shore D. Na tabela 3, abaixo, seguem os resultados obtidos:

\begin{tabular}{|l|l|}
\hline Formulações & Valores \\
\hline PLA & $70 D$ \\
\hline PLA10M & $75 D$ \\
\hline PLA15M & $77 D$ \\
\hline PLA20M & $73 D$ \\
\hline
\end{tabular}

Tabela 3: Resultado do ensaio de Dureza Shore D.

Fonte: Autores.

É possível perceber que o valor de dureza aumenta a medida que a concentração de carga é maior. Isto faz sentido uma vez que a madeira tende a ser mais dura que a matriz plástica. No entanto, este aumento não ocorre para o PLA + $20 \%$ de pó de madeira. Este fato pode ser atribuído à formação de aglomerados no material, gerando espaços vazios que comprometem as propriedades mecânicas do produto final. Petinakis (2009) determinou que a partir de $20 \%$ de pó de madeira, a interação entre as partículas de madeira aumenta de forma significativa, formando aglomerados, que geram zonas de concentração de estresse. Estes pontos possuem baixa resistência mecânica e formam regiões frágeis do material. Tais achados reforçam os resultados de dureza que foram obtidos e as observações constatadas.

\subsection{2 Índice de fluidez}

O resultado esperado é que a adição de carga diminua a fluidez do material, uma vez que a incorporação de partículas rígidas limita a mobilidade das cadeias, aumentando a viscosidade do material. Na tabela 4 a seguir, são apresentados os valores encontrados.

\begin{tabular}{|l|l|}
\hline Formulação & Valores \\
\hline PLA & $6,1 \mathrm{~g} / 10 \mathrm{~min}$ \\
\hline PLA10M & $8,6 \mathrm{~g} / 10 \mathrm{~min}$ \\
\hline PLA15M & $9,2 \mathrm{~g} / 10 \mathrm{~min}$ \\
\hline PLA20M & $9,6 \mathrm{~g} / 10 \mathrm{~min}$ \\
\hline
\end{tabular}

Tabela 4: Resultados do ensaio do Índice de Fluidez. Fonte: Autores.

Nota-se que este efeito não ocorreu no presente trabaIho. A fluidez do material aumentou com a adição de carga. Não foi encontrado na literatura caso semelhante. Os principais motivos pela formação de um material bem disperso e homogêneo são a afinidade entre carga e matriz e a capacidade de mistura do equipamento de processamento. Sabe-se que a madeira e o PLA não são totalmente compatíveis. Ademais, o equipamento usado possui canal de extrusão curto, suficiente apenas para aquecer o material e conformá-lo. A extrusora não possui uma zona de cisalhamento e mistura o que seria importante para misturar e homogeneizar a carga com o polímero.

Portanto, este resultado revela uma ineficiente dispersão da carga na matriz polimérica e da possível baixa afinidade química entre o pó de madeira e o PLA. Também, pode-se afirmar que a formação de espaços vazios ou microbolhas distorce o valor do ensaio e faz com que se encontrem valores diferentes do esperado na literatura.

\subsubsection{Microscopia óptica}

Abaixo na figura 5 são ilustradas as imagens geradas pelo ensaio de microscopia. Em todas as amostras é possível ver a formação de aglomerados e consequentemente de espaços vazios no material. A parte esbranquiçada das imagens trata-se da parte polimérica que não possui partículas de pó de madeira, provando que de fato a carga não se distribuiu bem pela matriz. Outro fato que é possível observar através da comparação entre as imagens, figura 5, é que a intensidade da coloração marrom se intensifica com o aumento de carga. A amostra PLA10M apresenta aspecto superficial e brilho do filamento de PLA puro. A partir da amostra PLA15M o filamento apresenta aspecto de madeira, evidenciado pelo aparecimento de porosidade superficial e coloração mais intensa. A imagem da amostra PLA20M prova a teoria apresentada nos demais resultados. A partir desta concentração, a amostra fica irregular, com muitos espaços vazios e muitos aglomerados. (TAO et al., 2017). 


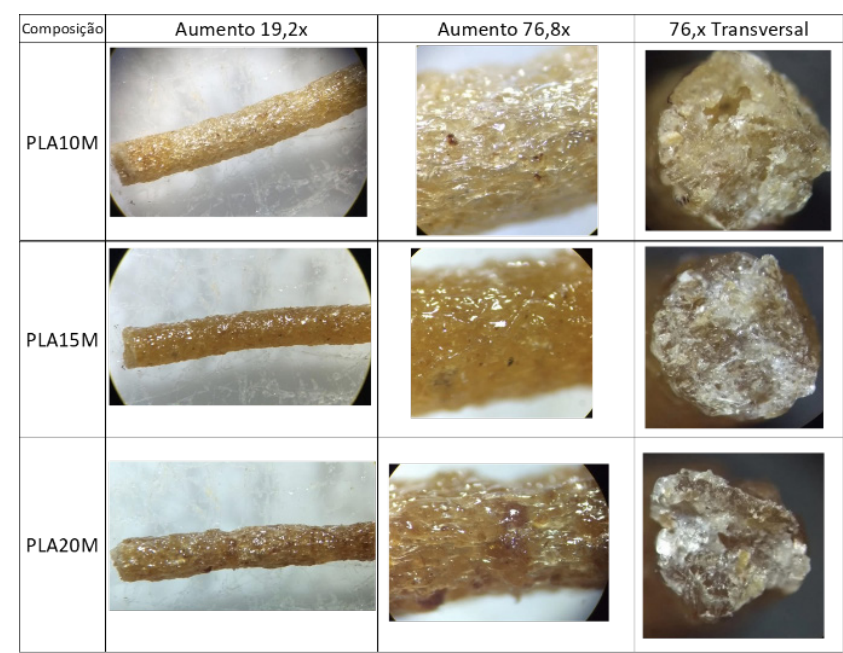

Figura 05: Imagens geradas pelo ensaio de microscopia. Fonte: Autores.

\subsubsection{Homogeneidade do filamento}

Os parâmetros de extrusão foram estabelecidos de maneira que o filamento tenha $\pm 1,75 \mathrm{~mm}$ de diâmetro. Uma aferição da variabilidade dimensional dos filamentos foi realizada ao longo de 2,0 m de filamento produzidos. $\mathrm{Na}$ tabela 5 abaixo é possível ver os valores de máxima e mínima, variância, desvio padrão e mediana.

\begin{tabular}{|l|l|l|l|l|}
\hline Valor & PLA & PLA10M & PLA15M & PLA20M \\
\hline $\begin{array}{l}\text { Máximo } \\
\text { (mm) }\end{array}$ & 1,7 & 1,92 & 2,08 & 1,95 \\
\hline $\begin{array}{l}\text { Mínimo } \\
\text { (mm) }\end{array}$ & 1,25 & 1,27 & 1,12 & 1,29 \\
\hline $\begin{array}{l}\text { Médio } \\
\text { (mm) }\end{array}$ & 1,48 & 1,51 & 1,61 & 1,52 \\
\hline $\begin{array}{l}\text { Variação } \\
\text { (\%) }\end{array}$ & $2 \%$ & $3 \%$ & $7 \%$ & $4 \%$ \\
\hline $\begin{array}{l}\text { Desvio } \\
\text { padrão }\end{array}$ & 0,129 & 0,176 & 0,257 & 0,197 \\
\hline
\end{tabular}

Tabela 5: Dados estatísticos das medições. Fonte: Autores.

É possível notar a heterogeneidade dos filamentos. Tal característica é atribuída a ausência de um sistema de bobinamento automatizado do filamento ao sair da extrusora. Esse dispositivo poderia controlar, assim, a espessura diametral dos filamentos ao saírem do bocal da extrusora uma vez que o puxamento constante do filamento tende a manter o diâmetro do filamento mais regular. De fato, os filamentos saíram de forma manual, sem haver o controle da velocidade de puxamento constante o que levou a variabilidade diametral de todos os filamentos produzidos. Outra constatação é o fato da maioria dos valores estarem abaixo de $1,70 \mathrm{~mm}$. Isto se deve a contração do material no resfriamento. Apesar de a variância ser menor que 10\%, ela é suficiente para dificultar o processo de alimentação na impressora 3D.

\subsubsection{Calorimetria exploratória diferencial (CED)} A partir dos dados obtidos no CED foi possível determinar tanto a temperatura de fusão do material e a temperatura de transição vítrea. Na figura 6 se encontra o gráfico gerado nesta análise. É possível notar que a temperatura de fusão do material aumenta com a adição de pó de madeira. Estas propriedades estão relacionadas com o grau de liberdade da cadeia polimérica. Quanto mais liberdade, as moléculas se movimentam mais facilmente, o que facilita a geração de aquecimento. A incorporação de fibras na matriz provoca pontos de impedimento nesta movimentação, fazendo com que o material necessite de mais energia para a mudança de estado. Contudo, verificou-se que a temperatura de transição vítrea da matriz PLA não foi sensivelmente alterada com a adição de resíduos de madeira o que se revela um fator positivo considerando que não há alteração nas propriedades termomecânicas da matriz e esse fator não afetaria o processo de impressão 3D.

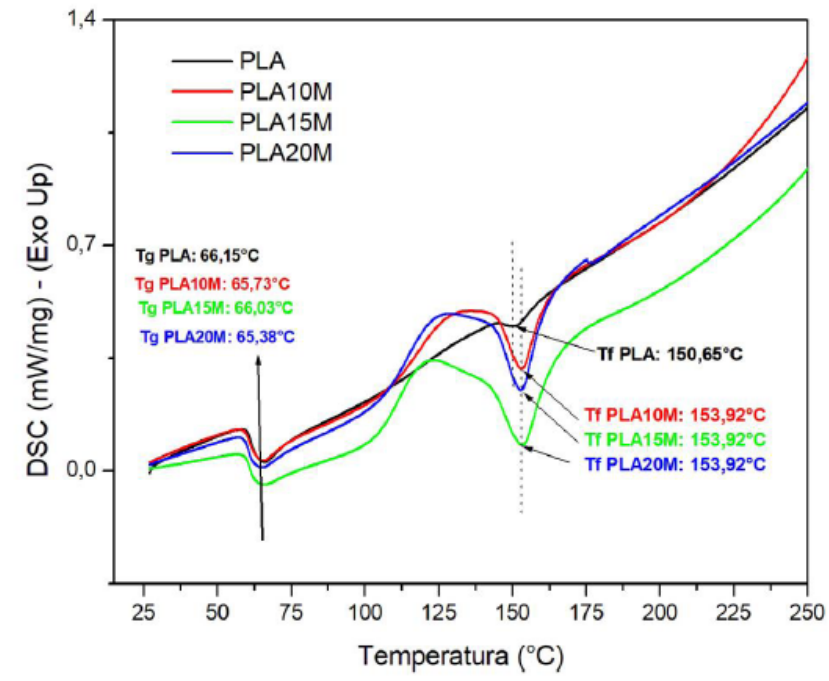

Figura 06: Análise por Calorimetria exploratória diferencial dos filamentos compósitos produzidos e da matriz de PLA pura. Fonte: Autores.

\subsection{Teste de impressão 3D}

A impressora possui um sistema de engrenagens que trabalha como um puxador, que leva o filamento até o canal de impressão, onde ele é fundido e impresso. No entanto, se constatou que os filamentos desenvolvidos possuem duas características que impedem que este processo ocorra de maneira eficaz:

i) O diâmetro heterogêneo do filamento fez com que a engrenagem não conseguisse puxar de maneira fluida. 
Quando as engrenagens encontram uma parte do filamento com diâmetro muito fino, não conseguem recoIher o material em direção ao bico de impressão e assim o material tranca naquele local.

ii) Os filamentos produzidos apresentam elevada fragilidade, quando comparados a filamentos comerciais de PLA usados na impressão 3D. Assim, percebeu-se uma certa dificuldade dos filamentos circularem pelo sistema de direcionamento de filamento da impressora 3D.

A solução encontrada foi utilizar uma impressora 3D que não possui este sistema de engrenagens e a inserção de filamento no canal de impressão é feito manualmente. Na figura 7 abaixo ao analisar o aspecto do corpo de prova, é possível notar que se trata de um material "printável", uma vez que apresenta estrutura homogênea e aspecto satisfatório. No entanto a fragilidade e a falta de homogeneidade impediram seu uso de maneira viável.

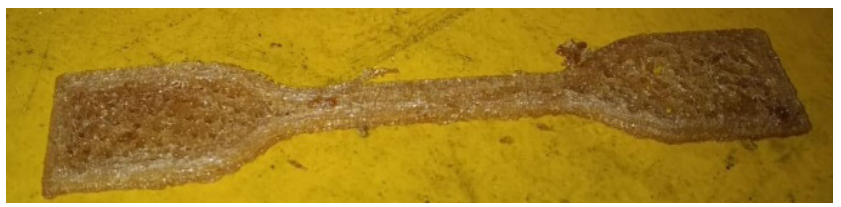

Figura 07: Teste de impressão 3D.

Fonte: Autores.

Sendo o intuito desta etapa a avaliação da "printabilidade" e a viabilidade de utilizar os filamentos desenvolvidos, verificou-se que tanto o filamento de PLA puro quanto os filamentos compósitos desenvolvidos tem potencial de impressão 3D mas precisam passar por aperfeiçoamento em termos de processo de mistura do resíduo de madeira bem como em termos do bobinamento dos filamentos produzidos para que a viabilidade de impressão 3D seja efetiva.

\section{CONSIDERAÇÕES FINAIS}

A partir da caracterização do pó de madeira conclui-se que este possui natureza química polar e superfície não porosa ou com presença de macro poros. Ambas as características dificultam a afinidade com a matriz polimérica. A polaridade é incompatível com a estrutura apolar do PLA e o perfil de porosidade impede uma ancoragem física entre os dois compostos. Viu-se também que o pó de madeira possui granulometria homogênea. $O$ fato das partículas estarem na escala de micrometros, aumenta a sua higroscopicidade. Isto é evidenciado pelo alto valor encontrado no ensaio de umidade e por isso é necessário fazer uma secagem previa. Esses achados indicam que um tratamento superficial para compatibilizar a matriz polimérica com as partículas de madeira se faz necessário.
No processo de confecção dos filamentos, as características do material e do processo dificultaram a obtenção de um filamento homogêneo. As variações no processamento fizeram com que a saída do material na matriz da extrusora oscilasse, gerando um filamento heterogêneo. Ainda, o fato de não ter um puxador automatizado acentuou esta característica, o que foi comprovado no ensaio de homogeneidade do diâmetro do filamento. Os demais ensaios como dureza, microscopia e fluidez provaram a teoria da formação de aglomerados e consequente formação de vazios. Isso foi revelado pela piora na resistência mecânica, em termos de dureza, com a adição de pó de madeira, deixando o material mais frágil e quebradiço. Estes espaços vazios, possivelmente, criam caminhos de propagação de falhas e redução nas propriedades mecânicas.

Ainda, o processo de extrusão no equipamento usado para realizar o filamento, principalmente em termos do perfil da rosa (tamanho e geometria) não favorecem um efetivo cisalhamento para misturar de forma mais plena as partículas de madeira na matriz PLA. Em extrusoras industriais, existem zonas de mistura e cisalhamento que podem forçar materiais de menor afinidade química a se misturarem fisicamente e formarem compósitos mais homogêneos. Agentes de compatibilização desse sistema também devem ser considerados.

Ao avaliar a "printabilidade" do material, viu-se que é possível imprimir peças com o filamento produzido. No entanto as características frágeis do material tornaram este processo inviável, uma vez que o mesmo se quebrava no sistema de alimentação do equipamento.

A partir da pesquisa desenvolvida, conclui-se que foi possível fabricar um filamento compósito de matriz PLA com reforço de fibras de madeira em pó com um aspecto visual que remete ao aspecto da madeira. Como a temática deste trabalho - materiais para impressão 3D - carece de mais trabalhos e espera-se que esta pesquisa estimule outros pesquisadores a aprofundar o tema para novos desenvolvimentos.

\section{AGRADECIMENTOS}

Este trabalho contou com apoio financeiro da FAPERGS por meio do Edital 01/2017 auxílio recém-doutor (ARD). Ao Laboratório de Biomateriais e Cerâmicas Avançadas da UFRGS.

\section{REFERÊNCIAS}

BARBOSA, A. P. Características estruturais e propriedades de compósitos poliméricos reforçados com fibras de buriti. 2011. Disponível em: http://uenf.br/ 
posgraduacao/engenharia-de-materiais/wp-content/ uploads/sites/2/2013/07/Tese-de-doutorado-_fibras-de-Buriti_.pdf. Acesso em: 04/10/2019.

BRENT, T.; LIU, Z.; FINKENSTADT, V.; LEWANDOWSKI, B.; OTT, S.; REIFSCHNEIDER, L. 3D printing biocomposites. 2015. Disponível em: $<10.2417 /$ spepro.005690>. Acesso em: 05/09/2019.

CHIA, H. N.; WE, M. B.; Recent advances in 3D printing of biomaterials. 2015. Disponível em <https:// doi.org/10.1186/s13036-015-0001-4> Acesso em: 03/03/2019.

FERNANDES, J. F. M. Estudo da Influência de Parâmetros de Impressão 3D nas Propriedades Mecânicas do PLA. 2016. Disponível em: <https://fenix.tecnico.ulisboa.pt/ downloadFile/281870113703681/Dissertacao_73521. pdf $>$. Acesso em: 18/04/2019.

FREITAS, J. C. Obtenção e caracterização de peças porosas de alumina obtidas por gelatinização. 2014.

GREENE, TIM. The Role of 3D Printing in Factory 4.0. IDC, Analize the future. White Paper. 2017.

KARIZ, M.; SERNEK, M. Effect of wood content in FDM filament on properties of 3D printed parts. 2018. Disponível em: <https://doi.org/10.1016/j.mtcomm.2017.12.016>. Acesso em: 03/09/2019

LE DUIGOUA, A.; CASTROB, M.; BEVANC, R.; MARTIN, N. 3D printing of wood fibre biocomposites: From mechanical to actuation functionality. 2016. Disponível em: $\quad<$ https://doi.org/10.1016/j.matdes.2016.02.018>. Acesso em: 22/09/2019

MORESCO, Mauro. Efeito de agente de acoplamento em compósitos de polipropileno com cargas vegetais. Trabalhos de Conclusão de Curso de Graduação. Universidade Federal do Rio Grande do Sul. Escola de Engenharia. Curso de Engenharia de Materiais. 2009. NATURE WORKS. Ingeo ${ }^{\text {TM }}$ Biopolymer 2003D Technical Data Sheet. Disponível em: https://www. natureworksllc.com/ /media/Technical_Resources/ Technical_Data_Sheets/TechnicalDataSheet_2003D_ FFP-FSW_pdf.pdf. Acesso em: 20/09/2019.

OLIVEIRA, B. M.; FILHO, J. M.; AFONSO, J. C. A densidade e a evolução do densímetro. 2013. Disponível em https://www.scienceopen.com/document?vid=cb623ba1-d2f1-4050-bc15-313392598f11. Acesso em 04/10/2019

PETINAKIS, E.; YU, L.; EDWARD, G.; DEAN, K.; LIU, H.; SCULLY, A. D. Effect of Matrix-Particle Interfacial Adhesion on the Mechanical Properties of Poly(lactic acid)/Wood-Flour Micro-Composites. 2009. Disponível em: <10.1007/s10924-009-0124-0>. Acesso em:
15/08/2019.

REIS, D. C. N. S. P. Importância do estudo da área superficial específica e porosidade do estearato de magnésio para o setor farmacêutico. 2013. Disponível em: <https://www.arca.fiocruz.br/handle/icict/7720>. Acesso em: 22/09/2019

ROCHA, M. C.G.; COUTINHO, F. M. B.; BALKE, S. Índice de Fluidez: Uma Variável de Controle de Processos de Degradação Controlada. De Polipropileno por Extrusão Reativa. Polímeros: Ciência e Tecnologia, vol.4, n3, p.33-37, 1994.

SCHUBERT, C.; VAN LANGEVELD, M. C.; DONOSO, L. A.; Innovations in 3D printing: a 3D overview from optics to organs. 2014. Disponível em: http://dx.doi. org/10.1136/bjophthalmol-2013-304446. Acesso em: 10/10/2019

TAO, Y.; WANG, H.; LI, Z.; LI, P. Development and Application of Wood Flour-Filled Polylactic Acid Composite Filament for 3D Printing. 2017. Disponível em: $<10.3390 /$ ma10040339>. Acesso em: 15/06/2019.

TEIXEIRA, M. G.; CESAR, S. F. Produção de compósito com resíduo de madeira no contexto da ecologia industrial. 2006. Disponível em: <http://madeira.set. eesc.usp.br/article/view/219> Acesso em: 05/11/2019 WANGA, X.; JIANGB M.; ZHOUB Z.; GOUA, J.; HUIC D. $3 D$ printing of polymer matrix composites: $A$ review and prospective. 2017. Disponível em: <https://doi. org/10.1016/j.compositesb.2016.11.034>. Acesso em: $16 / 09 / 2019$

ZHONG, R. Y.; XU, Xun.; KLOTZ, Eberhard.; NEWMAN, S. T. Intelligent Manufacturing in the Context of Industry 4.0: A Review. 2017. Disponível em <http://dx.doi.org/10.1016/J.ENG.2017.05.015>. Acesso em: 11/12/2018. 


\section{AUTORES}

ORCID: https://orcid.org/0000-0002-9559-3958

DANIEL LAUXEN SPOHR | Universidade do Vale do Rio dos Sinos (UNISINOS-RS) | Engenharia de Materiais | Dois Irmãos, RS - Brasil | Correspondência para: Endereço: rua Pastor Klein, 130, Dois Irmãos - Rio Grande do Sul, 93950-000 | E-mail: danielspohr13@gmail.com

ORCID: https://orcid.org/0000-0002-9065-9188

FELIPE ANTÔNIO LUCCA SÁNCHEZ, Dr. | Universidade do Vale do Rio dos Sinos (UNISINOS-RS) | Ciência e Tecnologia dos Materiais | Porto Alegre, RS - Brasil | Correspondência para: Av. Luiz Manoel Gonzaga, 744 - Bairro Três Figueiras Porto Alegre - RS, 90470-280| E-mail: felipe.lucsan@gmail.com

ORCID: https://orcid.org/0000-0001-7756-3117

ANDRÉ CANAL MARQUES, Dr. | Universidade do Vale do Rio dos Sinos (UNISINOS-RS) | Ciência e Tecnologia dos Materiais| Porto Alegre, RS - Brasil | Correspondência para: Av. Luiz Manoel Gonzaga, 744 - Bairro Três Figueiras Porto Alegre - RS, 90470-280| E-mail: andrecm@unisinos.br

\section{COMO CITAR ESTE ARTIGO}

SPOHR, Daniel Lauxen; SANCHEZ, Felipe Antônio Lucca; MARQUES, André Canal. Estudo Da Viabilidade De Fabricação De Filamentos Compósitos Sustentável Para Impressão 3D A Partir De Uma Matriz Pla Reforçada Com Resíduos De Fibras De Madeira. MIX Sustentável, [S.I.], v. 7, n. 4, p. 115-126, set. 2021. ISSN 24473073. Disponível em:<http://www.nexos. ufsc.br/index.php/mixsustentavel>. Acesso em: dia mês. ano. doi:https://doi.org/10.29183/2447-3073. MIX2021.v7.n4.115-126. 
